\title{
Mejora de la resistencia al desgaste de titanio y sus aleaciones utilizados para prótesis articulares
}

\author{
F.J. GIL, A.CRESPO, J.M.MANERO, D.RODRIGUEZ Y J.A.PLANELL \\ CREB. Departamento Ciencia de los Materiales e Ingeniería Metalúrgica. \\ Escuela Técnica Superior de Ingenieros Industriales de Barcelona.
}

Universidad Politécnica de Cataluña.

\begin{abstract}
Resumen
Se han estudiado los endurecimientos superficiales que se producen en la aleación Ti $6 \mathrm{Al} 4 \mathrm{~V}$, mediante los procesos de anodización electroquímica y mediante diferentes tratamientos térmicos a distintas microestructuras de dicha aleación, así como los procesos de nitruración mediante difusión gaseosa.. Los resultados de la combinación de la anodización y los tratamientos térmicos producen endurecimientos superiores a 1000 HV lo que mejorará la resistencia al desgaste de estas aleaciones en sus aplicaciones para prótesis articulares. Mejores resultados ofrecen los valores de nitruración que pueden alcanzar las 1400 unidades Vickers.
\end{abstract}

\section{Introducción}

Las aleaciones de titanio se comenzaron a utilizar en la década de los sesenta en aplicaciones de implantes articulares, debido a la elevada resistencia a la corrosión, buenas propiedades mecánicas y la excelente biocompatibilidad que presentan estos materiales frente al cuerpo humano (1). Esta adecuada respuesta in vivo parece ser debida a la compleja película de óxidos de titanio que se forma en la superficie del metal de manera espontánea cuando está en contacto con el medio ambiente; esta capa es de un espesor de $5 \mathrm{~mm}$ y consiste en $\mathrm{TiO}_{2}$, TiO y $\mathrm{Ti}_{2} \mathrm{O}_{3}$ que activa de capa pasivamente (2).

Sin embargo, su utilización como componente articular debe ser mejorada, ya que la baja resistencia al desgaste provoca la rotura de las capas pasivadas cuando el metal entra en fricción y hace que exista una aceleración en la corrosión y los residuos producidos se liberan al organismo. $(3,4)$

Por esta razón las aleaciones de titanio se deben tratar superficialmente para aumentar la dureza superficial, y así ser más resistentes al desgaste. En este trabajo se han estudiado los procesos de anodización electroquímica y diferentes tratamientos térmicos que aumentarán las durezas superficiales, además de estos incrementos se producen por la anodización electroquímica un descenso en la velocidad de corrosión de la aleación (5) y la resistencia mecánica entre el hueso y el implante aumenta en la interfaz produciendo una mayor estabilidad a largo plazo (6).

Existen diferentes procedimientos para aumentar la dureza superficial de las aleaciones de titanio.
De entre ellos el más sencillo es la anodización clásica que crea una capa de $\mathrm{TiO}_{2}$ con un espesor de $100 \mathrm{~nm}$. Este tratamiento representa una mejora respecto a la dureza del titanio no tratado, pero la capa es frágil y no puede soportar elevados esfuerzos mecánicos.

Las deposiciones química y electroquímica de cromo o niquel y la técnica del PVD (deposición física en fase vapor) son otras técnicas utilizadas de endurecimiento consiguiendo capas de TiN de espesores de 1 a $10 \mathrm{~mm}$. Estas capas logran aumentar los coeficientes de resistencia al desgaste cuatro veces referido al titanio sin tratamiento. Estas técnicas de deposición pueden ser generadas por evaporación con ayuda de un haz de electrones, pulverización catódica en corriente continua, pulverización catódica con aplicación de campos magnéticos y pulverización catódica a alta frecuencia o por plasma $(7,8)$.

Otro tipo de tratamientos son los denominados termoquímicos que se realizan mediante difusión en estado sólido de carbono y nitrógeno al sustrato, estableciendo enlaces químicos entre el metal base y la capa endurecida, lo que provoca un revestimiento más homogéneo y con mejores propiedades mecánicas de la interfacie metal capa. Los procesos CVD (deposisicón química en fase de vapor), baños de nitruración de sales, proporcionan unos espesores de capa endurecida de 10 a $100 \mathrm{~mm}$., obteniendo unos valores de dureza de 750 a 800 $\mathrm{HV}$, aumentando la resistencia al desgaste en un factor de $10 \mathrm{y}$ el coeficiente de fricción pasa de 0.32 a 0.18 . 
Sin embargo, los procesos termoquímicos no son ampliamente utilizados ya que los tratamientos para que ocurra la difusión de $\mathrm{C}$ y $\mathrm{N}$ deben realizarse a una temperatura de $800^{\circ} \mathrm{C}$ lo que produce que otros elementos como el oxígeno y el hidrógeno por los que tiene gran avidez el titanio se incorporen a la microestructura produciendo pasivaciones, fragilidad e impiden que se incorporen el C y el N (9 10).

La nitruración iónica de la aleación Ti $6 \mathrm{Al} 4 \mathrm{~V}$, puede alcanzar profundidades desde 10 a $100 \mathrm{~mm}$. según el tiempo y la temperatura de tratamiento. Las durezas superficiales pueden alcanzar los 1500 $\mathrm{HV}$, lo que multiplica por tres el coeficiente de resistencia al desgaste cuando lo comparamos con las deposiciones químicas o electroquímicas (11 12).

En este trabajo, se han estudiado los endurecimientos producidos por diferentes tratamientos térmicos de la aleación Ti $6 \mathrm{Al} 4 \mathrm{~V}$, estudiándose en la estructura martensítica el aumento de dureza producidos por diferentes tratamientos de revenido. Asimismo se han estudiado los aumentos de dureza que se obtienen en los procesos de anodizado estudiando la influencia de los potenciales aplicados y la microestructura del material. Por último, se ha intentado optimizar la microestructura, la anodización y los tratamientos térmicos para conseguir la máxima dureza. Estas técnicas de endurecimiento son más sencillas, versátiles y en las que se requieren como veremos pequeños tiempos de tratamiento tanto en los térmicos como en los de anodización. También describiremos los procesos de nitruración y podremos apreciar como los bruscos endurecimientos provocan una resistencia al desgaste muy importante que tendrán interesantes aplicaciones tecnológicas.

\section{Material y método experimental}

La aleación Ti $6 \mathrm{Al} 4 \mathrm{~V}$ utilizada en el presente trabajo, presenta una composición química con bajos contenidos en elementos intersticiales, que se muestra en la Tabla I. La microestructura de recepción ha sido la correspondiente a la mill annealed totalmente recocida, cumpliendo la norma ASTM F136 84 para el Ti 6Al 4V forjado para aplicaciones quirúrgicas. Esta microestructura se puede observar en la Figura 1.

\section{Tratamientos térmicos}

Se cortaron muestras cilíndricas de $12 \mathrm{~mm}$. de diámetro y $4 \mathrm{~mm}$. de altura para estudiar las durezas que se consiguen con cuatro microestructuras de esta aleación.
La primera de ellas es la microestructura de recepción mill annealed que proviene de forja con un recocido posterior de 2 horas a $600^{\circ} \mathrm{C}$ en la región $\alpha$ $+\beta$. Se obtiene una microestructura de granos equiaxiales de fase $\alpha$, en una matriz de fase $\beta$ que, en el enfriamiento lento posterior, se transformará en una mezcla $\alpha+\beta$ de morfología de placas $\alpha$ de Widmanstatten rodeadas de fase $\beta$.

La segunda microestructura es la denominada de Widmanstatten y se ha obtenido calentando las muestras a temperaturas superiores a la $\beta$ transus $\left(1050^{\circ} \mathrm{C}\right)$ durante una hora y posterior enfriamiento lento en el interior del horno. Este tratamiento térmico se ha llevado a cabo en atmósfera controlada de Argón. La morfología obtenida es de granos gruesos formadas por placas $\alpha$ de Widmanstatten empaquetadas, en una matriz de fase $\beta(13,14)$. Esta microestructura se puede apreciar en la Figura 2.

La tercera microestructura es la denominada martensítica $\alpha$ ' obteniéndose con el tratamiento térmico comentado en la microestructura anterior, pero con enfriamiento brusco (temple en agua a $20^{\circ} \mathrm{C}$ ) desde $1050^{\circ} \mathrm{C}$ con la fase $\beta$ estable. La microestructura obtenida es en forma de agujas muy finas, formando colonias de placas paralelas con alta densidad de dislocaciones, esta puede observarse en la Figura 3. $(14,15)$.

La cuarta microestructura estudiada es la compuesta por fase $\alpha$ y de matriz $\alpha$ '. Esta a diferencia de la anterior la solución no se realiza el tratamiento térmico a temperaturas superiores de la $\beta$ transus sino que la solución se realiza en el campo $\alpha+\beta$, a una temperatura de $950^{\circ} \mathrm{C}$ durante una hora. A esta temperatura hay fase $\alpha \sin$ transformar y la matriz de fase $\beta$, la proporción de las fases viene regida por la ley de la palanca, al templar desde la región $\alpha+\beta$, la fase $\alpha$ no es susceptible de transformación martensítica pero sí la fase $\beta$, obteniéndose una mezcla de fase $\alpha, \alpha$ y $\beta$ retenida que no ha transformado (Figura 4). (16).

La fase $\beta$ retenida se denomina metaestable y está presente en la microestructura debido a no $\alpha$ es rica $\beta$ estabilizador y hace disminuir la temperatura $M_{s}$, por tanto al enfríar bruscamente, parte de la fase $\beta$ transforma y otra queda retenida ya que no se alcanza la temperatura $\mathrm{M}_{\mathrm{f}^{\prime}}$

Estas dos últimas microestructuras se sometieron a posteriores tratamientos térmicos de revenido y maduración, en el primer caso a temperaturas de 400,700 y $800^{\circ} \mathrm{C}$ y en el segundo caso a $700^{\circ} \mathrm{C}$ durante diferentes tiempos de tratamiento con temple posterior en agua a $20^{\circ} \mathrm{C}$. 


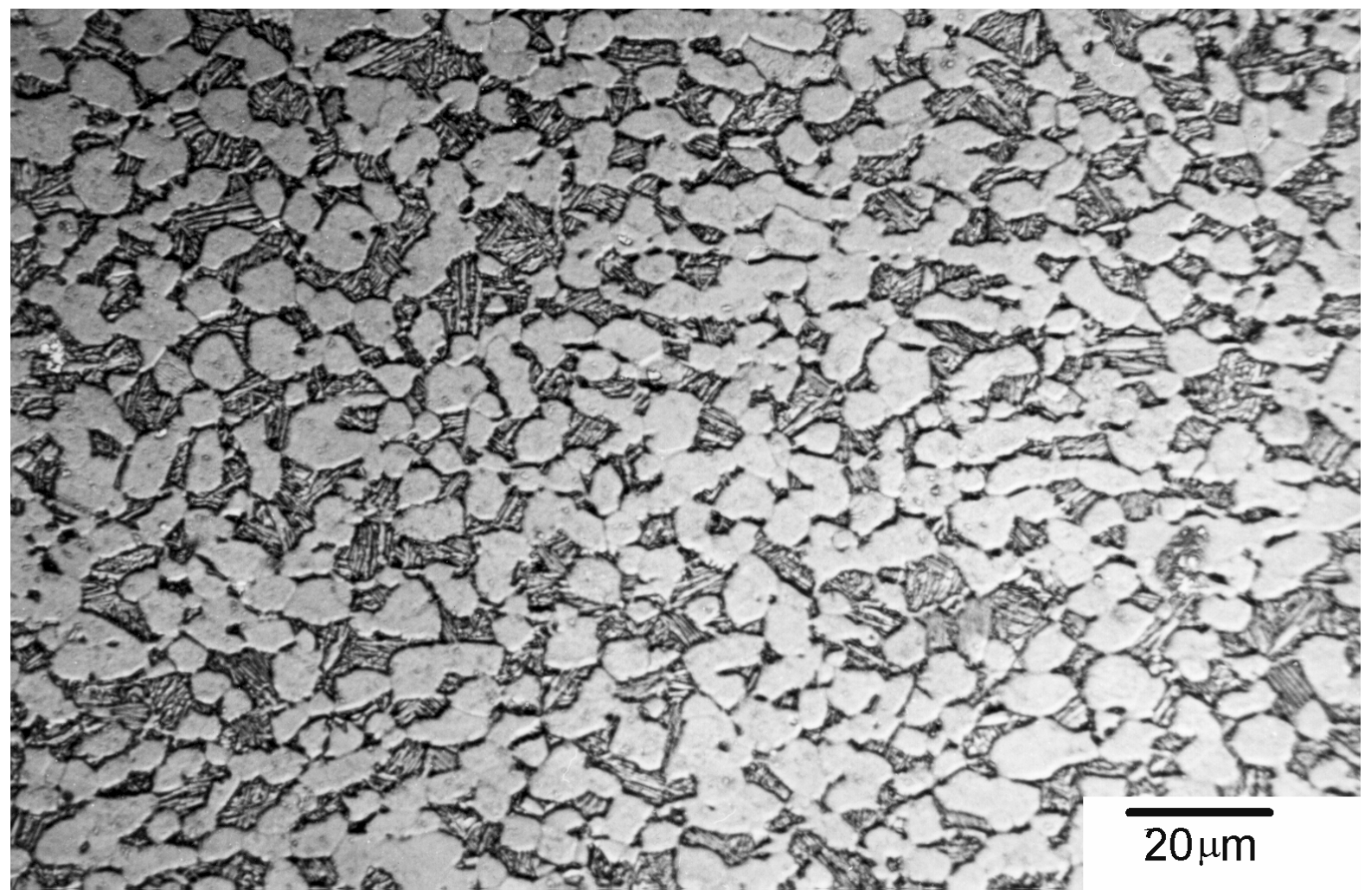

Figura 1. Estructura Mill annelaed.

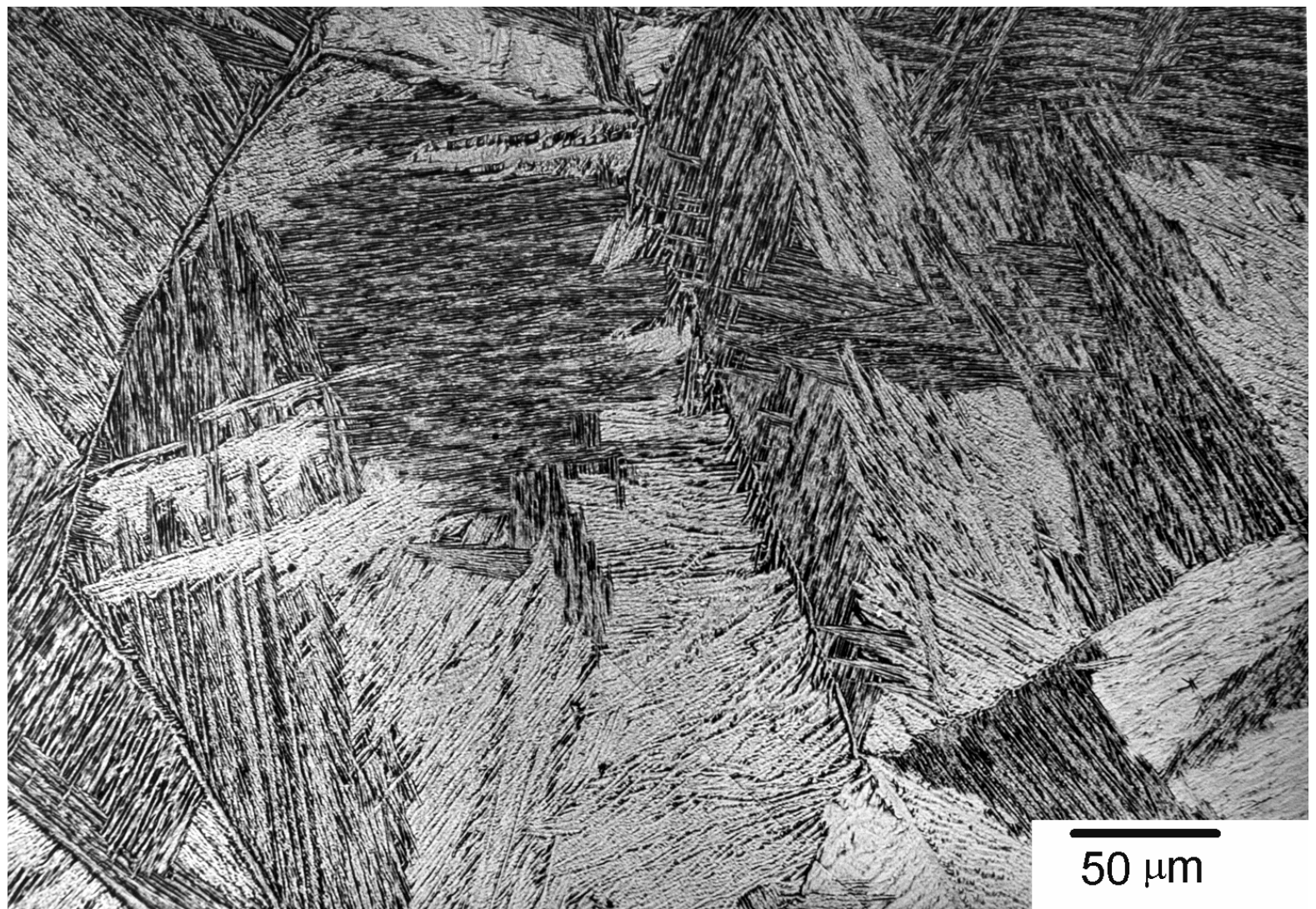

Figura 2. Estructura Widmasntatten. 


\section{Tratamiento de anodización}

El proceso electrolítico se realiza con una solución acuosa de sulfatos amónico y magnésico, ortofosfato sódico y ácido sulfúrico con ánodo de acero inoxidable.

La anodización se realiza a diferentes potenciales manteniéndose el tiempo constante a $10 \mathrm{~s}$, obteniéndose capas de óxidos de diferentes estequiometrías y grosores. El espesor de estas capas fué determinado por un medidor de espesores de alta sensibilidad mediante la aplicación de corrientes de Foucault.
Estos tratamientos se han realizado a probetas pulidas de las cuatro microestructuras anteriormente citadas y en las correspondientes martensíticas $\alpha^{\prime}$ y $\alpha+\alpha^{\prime}+\beta$ se realizaron posteriores tratamientos de revenido, maduración y de difusión de oxígeno de la capa anodizada al interior de la aleación.

Para cada muestra se determinaron la dureza Vickers aplicando $1000 \mathrm{~g}$. de carga durante $15 \mathrm{~s}$. Los valores que se expresan en las Tablas de resultados es la media aritmética de 10 indentaciones.

Tabla I. Composición química en porcentajes de peso de la aleación estudiada.

\begin{tabular}{||l|l|l|l|l|l|l||}
\hline \hline $\mathrm{Al}$ & $\mathrm{V}$ & $\mathrm{Fe}$ & $\mathrm{C}$ & $\mathrm{O}_{2}$ & $\mathrm{~N}_{2}$ & $\mathrm{H}_{2}$ \\
\hline 6.1 & 4.0 & 0.11 & 0.021 & 0.09 & 0.010 & 0.003 \\
\hline
\end{tabular}

Tabla II. Temperaturas y tiempos de los tratamientos realizados.

\begin{tabular}{|c||c|c|c|c|}
\hline \multicolumn{1}{|c||}{$\mathrm{T}\left[{ }^{\circ} \mathrm{C}\right]$} & \multicolumn{4}{c|}{ Tiempo (horas) } \\
\hline \hline 700 & 1 & 4 & 8 & 16 \\
\hline 900 & 1 & 4 & 8 & 16 \\
\hline 950 & 1 & 4 & 8 & 16 \\
\hline
\end{tabular}

Tabla III. Resultados del tratamiento de anodización para la microestructura mill annealed.

\begin{tabular}{||l|l|l|l|l||}
\hline \hline Probeta & $\begin{array}{l}\text { Voltaje } \\
\text { V }\end{array}$ & Color & Espesor nm & Dureza HV \\
\hline 0 & 0 & Metálico & - & 280 \\
\hline 1 & 10 & Dorado & 30 & 313 \\
\hline 2 & 15 & Ocre & 40 & 340 \\
\hline 3 & 20 & Azul & 70 & 356 \\
\hline 4 & 25 & Azul & 90 & 361 \\
\hline 5 & 30 & Azul & 120 & 369 \\
\hline 6 & 35 & Verde & 150 & 372 \\
\hline 7 & 40 & Verde & 170 & 375 \\
\hline 8 & 45 & Verde & 240 & 380 \\
\hline 9 & 50 & Amarillo & 290 & 383 \\
\hline 10 & 55 & Amarillo & 320 & 385 \\
\hline 11 & 60 & Violeta & 420 & 393 \\
\hline \hline
\end{tabular}


Para las muestras que dan máximos valores de dureza se estudiaron mediante un nanoindentador controlado por ordenador. Este equipo estaba situado en una habitación aislada de vibraciones y a una temperatura constante de $25^{\circ} \mathrm{C}$. El indentador es Vickers y la carga que se aplica es de $20 \mathrm{mN}$ durante 30 s., determinándose los perfiles de dureza respecto a la profundidad de la capa y las propiedades elásticas de la misma (17).

\section{Tratamiento de nitruración mediante difusión gaseosa}

Se estudiaron rótulas de titanio grado 3 de la empresa Klockner, en las que se han realizado 12 tratamientos térmicos, con diferentes temperaturas y tiempos, los cuales se detallan en la Tabla II.

Las probetas, una vez tratadas, se dejan enfriar en horno, a fin de obtener una estructura de granos alfa, que permita compensar el aumento de fragilidad producido por la nitruración.

Los experimentos se realizaron en un horno tubular, con tubo de alúmina, capaz de alcanzar los $1300^{\circ} \mathrm{C}$. Para conseguir una atmósfera de nitrógeno, se conectó al cilindro de alúmina una bombona de nitrógeno de alta pureza, y se mantuvo cerrado el tubo durante los experimentos. Un esquema del mismo se puede apreciar en la Figura 5.

Una vez se ha realizado el tratamiento, se prepara la probeta para observar la microestructura y realizar las mediciones de dureza y desgaste. Los ensayos de dureza se realizaron con un microdurómetro Matzsuzawa de alta precisión aplicando a la superficie una carga de $1 \mathrm{Kg}$ durante 15 segundos. Los ensayos de desgaste se realizaron siguiendo la norma ASTM de ensayos adhesivos pin-on-disk en medio salivar a $37^{\circ} \mathrm{C}$ aplicando la carga de $25 \mathrm{Kg}$. A medida que se realizaba el ensayo de desgaste se controlaban las medidas gravimétricas para determinar las pérdidas de peso con el tiempo mediante una balanza de alta precisión.

\section{Resultados experimentales y dsicusión}

Los resultados de microdurezas después del tratamiento de anodización se muestran en las Tablas III,IV, V y VI correspondientes a las microestructuras de mill annealed, Widmanstatten, martensítica y la correspondiente a las fases $\alpha+\alpha$ ' $+\beta$, respectivamente.

Se puede apreciar que la variación de los tiempos de aplicación de una diferencia de potencial constante no ejerce una influencia tan importante como el voltaje en los valores de dureza superficial. Los tratamientos de anodización producen aumentos de unas cien unidades Vickers para cada microestructura en las condiciones de $60 \mathrm{~V}$ de diferencia de potencial y diez segundos en el tiempo de aplicación. Las durezas, como se desprende de los valores de las Tablas aumentan a mayor potencial aplicado, es decir a mayores grados de oxidación del titanio. Si relacionamos el valor de dureza de la microestructura mill annealed con el máximo obtenido para la microestructura con fases $\alpha+\alpha^{\prime}+\beta$ se obtienen valores del doble de la dureza original.

Además de los aumentos de dureza que provocan las capas de óxido formadas, las capas anodizadas producen un descenso en la liberación de iones metálicos de titanio, aluminio y vanadio, decreciendo los riesgos de la irritación local o metalosis provocado por la difusión de iones metálicos en los tejidos circundantes $(6,18,19)$.

De las cuatro microestructuras estudiadas la martensítica y la tratada en la región $\alpha+\beta$ son las que proporcionan mayores valores de dureza. Estas dos microestructuras son susceptibles de aumentar sus durezas realizando tratamientos térmicos de revenido para la martensita y de maduración para la fase $\beta$.

Los diagramas TTT y CCT para la aleación Ti $6 \mathrm{Al} 4 \mathrm{~V}$ no están bien caracterizados hasta el momento y las temperaturas $M_{s}$ y $M_{f}$ que marcan el inicio y fin de la transformación martensítica no están todavía claramente definidas (20) (21). Sin embargo, hay trabajos $(22,23)$ que muestran mediante dilatometría que la temperatura $\mathrm{M}_{\mathrm{f}}$ para la aleación Ti $6 \mathrm{Al} 4 \mathrm{~V}$ es cercana a los $600^{\circ} \mathrm{C}$ y por tanto la microestructura martensítica $\alpha$ ' obtenida no tendrá $\beta$ retenida. Por tanto el tratamiento térmico será únicamente de revenido.

Los resultados de dureza respecto al tiempo de revenido se observan en la Figura 1. Las formas de las curvas de endurecimiento son características de los procesos de nucleación y crecimiento. Se aprecia para los revenidos a $800^{\circ} \mathrm{C}$ un brusco aumento de los valores de dureza hasta unos 20 minutos de tratamiento, para posteriormente estabilizarse a valores de unos $410 \mathrm{HV}$. Este endurecimiento se produce por la descomposición de la martensita $\alpha$ ' en un fino precipitado de $\alpha$ en los bordes de las agujas de martensita y la formación de fase $\beta$, como se puede observar en la Figura $7(24,25)$. El proceso que tiene lugar

$$
\alpha^{\prime} \rightarrow \alpha+\beta .
$$




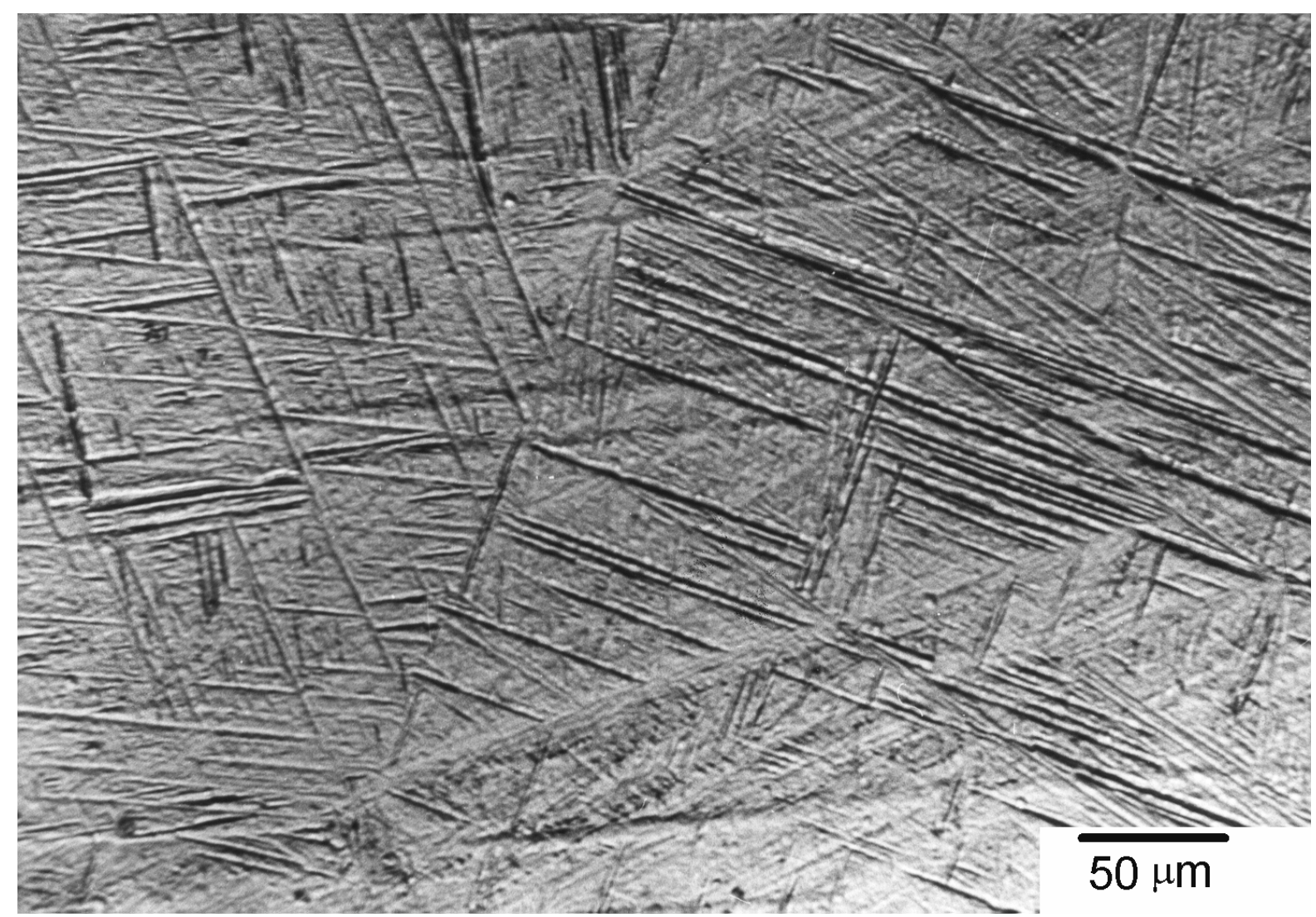

Figura 3. Estructura martensítica.

Tabla IV. Resultados de anodización para la microestructura Widmanstatten.

\begin{tabular}{||l|l|l|l|l||}
\hline Probeta & $\begin{array}{l}\text { Voltaje } \\
\text { V }\end{array}$ & Color & Espesor nm & Dureza HV \\
\hline 0 & 0 & Metálico & - & 319 \\
\hline 1 & 10 & Dorado & 20 & 335 \\
\hline 2 & 15 & Ocre & 30 & 360 \\
\hline 3 & 20 & Azul & 70 & 370 \\
\hline 4 & 25 & Azul & 80 & 375 \\
\hline 5 & 30 & Azul & 80 & 390 \\
\hline 6 & 35 & Verde & 140 & 392 \\
\hline 7 & 40 & Verde & 140 & 400 \\
\hline 8 & 45 & Verde & 180 & 410 \\
\hline 9 & 50 & Amarillo & 220 & 412 \\
\hline 10 & 55 & Amarillo & 240 & 415 \\
\hline 11 & 60 & Violeta & 360 & 421 \\
\hline \hline
\end{tabular}


Tabla V. Resultados de anodización para la microestructura martensítica.

\begin{tabular}{||c|l|l|l|l||}
\hline Probeta & $\begin{array}{l}\text { Voltaje } \\
\text { V }\end{array}$ & Color & Espesor nm & Dureza HV \\
\hline 0 & 0 & Metálico & - & 330 \\
\hline 1 & 10 & Dorado & 30 & 430 \\
\hline 2 & 15 & Ocre & 30 & 440 \\
\hline 3 & 20 & Azul & 70 & 440 \\
\hline 4 & 25 & Azul & 80 & 455 \\
\hline 5 & 30 & Azul & 80 & 442 \\
\hline 6 & 35 & Verde & 140 & 445 \\
\hline 7 & 40 & Verde & 160 & 445 \\
\hline 8 & 45 & Verde & 180 & 443 \\
\hline 9 & 50 & Amarillo & 250 & 460 \\
\hline 10 & 55 & Amarillo & 260 & 460 \\
\hline 11 & 60 & Violeta & 500 & 455 \\
\hline \hline
\end{tabular}

Tabla VI. Resultados del tratamiento de anodización para la microestructura $a+a$ ’ $+\beta$.

\begin{tabular}{||c|l|l|l|l||}
\hline Probeta & $\begin{array}{l}\text { Voltaje } \\
\text { V }\end{array}$ & Color & Espesor nm & Dureza HV \\
\hline 0 & 0 & Metálico & - & 390 \\
\hline 1 & 10 & Ocre & 30 & 401 \\
\hline 2 & 15 & Azul & 60 & 410 \\
\hline 3 & 20 & Azul & 70 & 410 \\
\hline 4 & 25 & Azul & 80 & 430 \\
\hline 5 & 30 & Verde & 120 & 450 \\
\hline 6 & 35 & Verde & 140 & 450 \\
\hline 7 & 40 & Verde & 190 & 460 \\
\hline 8 & 45 & Amarillo & 270 & 480 \\
\hline 9 & 50 & Amarillo & 280 & 480 \\
\hline 10 & 55 & Violeta & 420 & 500 \\
\hline 11 & 60 & Violeta & 510 & 510 \\
\hline \hline
\end{tabular}


La descomposición de la martensita al ser un proceso gobernado por la difusión en estado sólido depende de la temperatura, como se observa en la Figura 6 a mayor temperatura se consigue la máxima dureza en un menor tiempo de tratamiento térmico. Para la temperatura de revenido de $400^{\circ} \mathrm{C}$ se obtienen durezas máximas de $350 \mathrm{HV}$ ya que esta temperatura no es suficiente para activar el proceso de descomposición de las placas martensíticas.

Las microestructuras obtenidas por temple desde la zona $\alpha+\beta$ están formadas por granos de $\alpha$ (no transformados en el calentamiento), en una matriz de martensita y $\beta$ metaestable. Esta matriz difiere de la obtenida por temple desde $\beta$ en que la $\beta$ residual será de mayor contenido en elemento estabilizador de $\beta$ y podrá haber mayor proporción de $\beta$, lo que ofrecerá ventajas de cara al endurecimiento posterior.

Este aumento de dureza vendrá dado por dos tipos de transformación, el revenido o descomposición de la martensita presente en la microestructura $\left(\alpha^{\prime} \rightarrow \alpha\right.$ $+\beta)$ y la maduración de la fase $\beta$ que denominamos metaestable.

La transformación que tiene lugar es:

$$
\beta \rightarrow \beta+\mathrm{w} \rightarrow \beta+\alpha
$$

la fase metaestable transforma a las fases de equilibrio termodinámico $\alpha+\beta$. Esta descomposición se realiza sólo a relativamente altas temperaturas dado que, aunque sea metaestable, la nucleación en $\beta$ de la fase $\alpha$ es dificil. En ocasiones puede aparecer la etapa intermedia $(\beta+w)$ normalmente no suele formarse en aleaciones con un mínimo de $3 \%$ de aluminio (26)(27). La fase w es un precipitado coherente, en forma de cubos, muy finamente disperso y muy abundante. Esta fase causa una fragilidad acusada y hay que evitar su formación mediante calentamientos a temperaturas superiores a $\operatorname{los} 500^{\circ} \mathrm{C}(28)$.

En la Figura 8 se muestra la representación de dureza respecto al tiempo de tratamiento térmico (revenido y maduración), se aprecia que se alcanzan valores de dureza máximos de $616 \mathrm{HV}$, más de doscientas unidades respecto a la estructura obtenida después del temple desde la región $\alpha+\beta$ y más de trescientas unidades respecto a la microestructura mill annealed de recepción.

La Figura 8 muestra un tipo de curva similar a la de revenido (Figura 7), se observa una estabilización de las durezas a partir de un tiempo de tratamiento de 120 minutos. A partir de estos tiempos la dureza aumenta ligeramente y no se observan globulizaciones de precipitados de $\alpha$ que indicarían un sobrerevenido o sobremaduración haciendo descender dichos valores.

Una vez estudiados los resultados de los procesos de anodización y de tratamientos térmicos por separado, se ha estudiado una combinación entre el proceso de anodización y de tratamiento térmico con el fin de optimizar los valores de dureza superficial.

Se ha escogido la microestructura tratada en la región $\alpha+\beta$ y se ha realizado un anodizado electroquímico aplicando una diferencia de potencial de $50 \mathrm{~V}$ durante $60 \mathrm{~s}$. Posteriormente, se realizaron tratamientos térmicos a $700^{\circ} \mathrm{C}$ durante diferentes tiempos y enfriamiento en agua a $20^{\circ} \mathrm{C}$. Este tratamiento térmico producirá el revenido de la martensita $\alpha$ ', la maduración de la fase $\beta$ metaestable y la incorporación controlada de oxígenos desde la capa anodizada al substrato del metal. Este último mecanismo produce aumentos importantes en la resistencia mecánica y dureza del material; tan solo un $0.2 \%$ de oxígeno eleva en $300 \mathrm{MPa}$ los valores de resistencia y se produce una disminución del $10 \%$ en el alargamiento $(29,30,31)$.

La microestructura que se obtiene es de granos poliédricos de fase $\alpha$, en la zona superficial, ya que el oxígeno es un elemento $\alpha$ estabilizador. A medida que nos distanciamos de la superficie la densidad de granos $\alpha$ va disminuyendo y van apareciendo en la microestructura agujas de martensitas descompuestas en finos precipitados de $\alpha$.

En la Tabla VII se muestran los valores máximos de dureza, energias elástica, plástica y total y la relación entre el módulo elástico y el coeficiente de Poisson. En la Figura 3 se muestra la variación de la dureza respecto a la profundidad de la capa endurecida. Como se desprende de los valores de la Tabla VII, referente a la energía elástica y plástica, corresponden aproximadamente al $50 \%$ de la total cada una de ellas y esto indica que la capa no es quebradiza y que puede soportar esfuerzos mecánicos.

El valor máximo de dureza corresponde a 1322 HV para un tiempo de 120 minutos.

En la Figura 9 se muestra la variación de la dureza respecto a la profundidad de la capa desde la superficie. Se aprecia que los valores máximos de dureza corresponden hasta $5 \mu \mathrm{m}$ donde la proporción de fase $\alpha$ es máxima y tiene un contenido de oxígeno más elevado. Estos valores de dureza van disminuyendo gradualmente, es a distancias de más de $120 \mathrm{~mm}$ donde la dureza es de $390 \mathrm{HV}$ correspondiente a la microestructura sin tratar superficialmente.

En la actualidad, el tratamiento de superficies para endurecimiento más comúnmente utilizado es la implantación iónica debido a las grandes ventajas que 
Tabla VII. Dureza $(H)$, energía elástica $\left(W_{\mathrm{e}}\right)$, energía plástica $\left(\mathrm{W}_{\mathrm{p}}\right)$, energía total $\left(\mathrm{W}_{\mathrm{T}}\right)$ y la relación E/1- $\mathrm{n}^{2}$ ) donde E es el módulo de Young y $\mathrm{n}$ es el coeficiente de Poisson correspondientes a las muestras tratadas a $700^{\circ} \mathrm{C}$ durante diferentes tiempos.

\begin{tabular}{||c|c|c|c|c|c||}
\hline $\begin{array}{c}\text { Tiempo } \\
(\mathrm{min})\end{array}$ & $\mathrm{H}(\mathrm{HV})$ & $\begin{array}{c}\mathrm{W}_{\mathrm{e}} \\
(\mathrm{nJ})\end{array}$ & $\begin{array}{c}\mathrm{W}_{\mathrm{p}} \\
(\mathrm{nJ})\end{array}$ & $\begin{array}{c}\mathrm{W}_{\mathrm{T}} \\
(\mathrm{nJ})\end{array}$ & $\begin{array}{c}\mathrm{E} / 1-v^{2} \\
(\mathrm{GPa})\end{array}$ \\
\hline 60 & 1005 & 0.96 & 1.04 & 2.00 & 232 \\
\hline 120 & 1322 & 1.09 & 1.06 & 2.16 & 229 \\
\hline 600 & 1250 & 0.92 & 0.90 & 1.82 & 258 \\
\hline 1200 & 908 & 0.89 & 1.07 & 1.96 & 250 \\
\hline
\end{tabular}

Tabla VIII. Valores de dureza superficial de las probetas tratadas, en $\mathrm{HV}_{500}$.

\begin{tabular}{|c||c|c|c|c|}
\hline \multicolumn{1}{|c||}{} & \multicolumn{4}{c|}{$\mathrm{t}[\mathrm{h}]$} \\
\hline $\mathrm{T}\left[{ }^{\circ} \mathrm{C}\right]$ & 1 & 4 & 8 & 16 \\
\hline \hline 700 & 413 & 544 & 717 & 547 \\
\hline 900 & 841 & 895 & 928 & 1074 \\
\hline 950 & 1018 & 1028 & 967 & 1097 \\
\hline
\end{tabular}

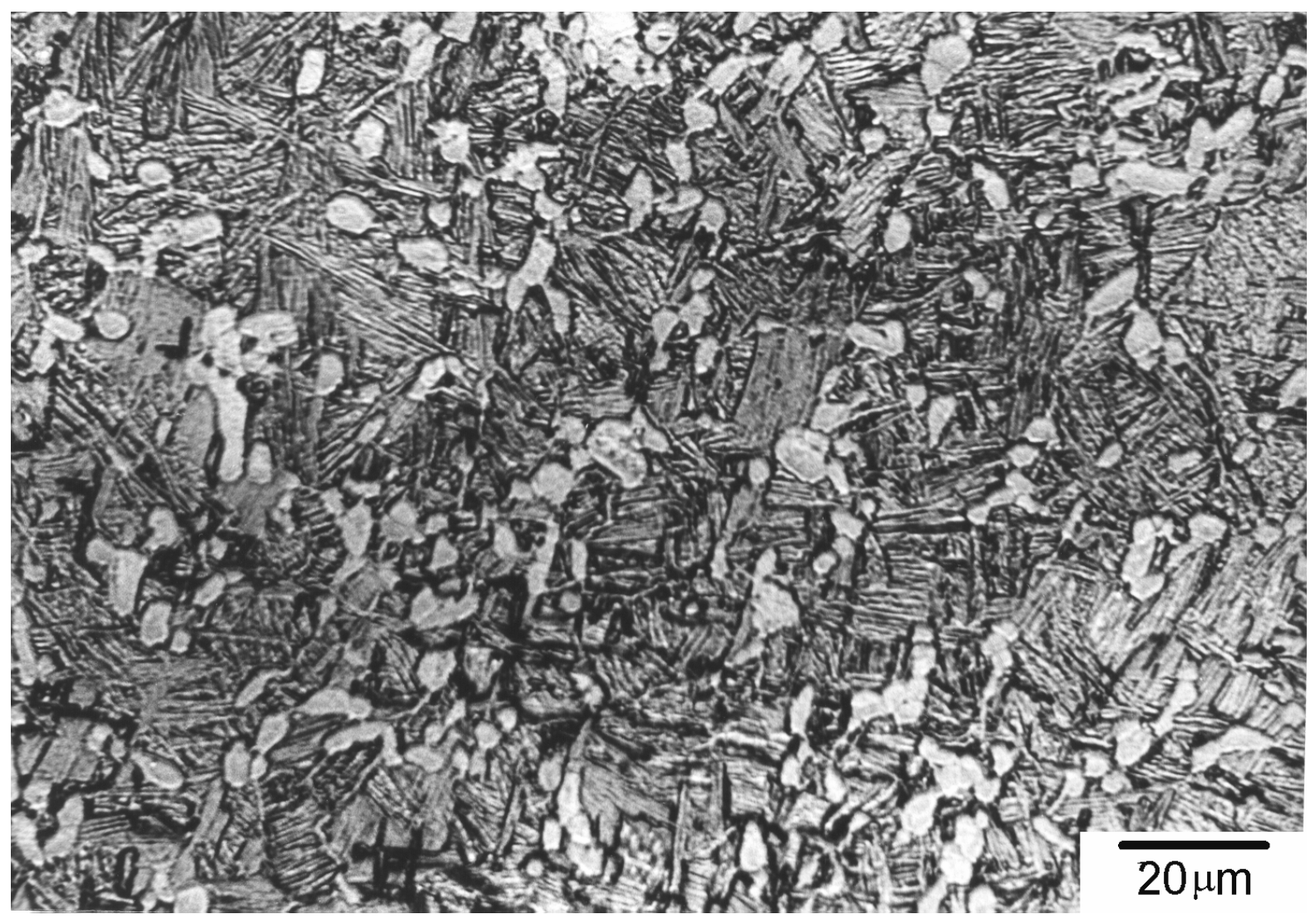

Figura 4. Estructura con presencia de fases a, a' y b retenida. 
posee $(32,=33)$ : cualquier elemento puede ser implantado independientemente de la solubilidad y del equilibrio termodinámico, pequeño calentamiento del substrato, no hay cambios dimensionales no hay problemas con la adhesión, reduce la corrosión y un aumento en la resistencia a fatiga.

Sin embargo, la implatación iónica produce una transición muy brusca de dureza y las técnicas de implantación son muy caras. Con este nuevo proceso de endurecimiento se alcanzan valores de dureza casi del mismo orden que la técnica de implantación iónica para el titanio y con las mismas propiedades, sin embargo estas nuevas técnicas de endurecimiento son más sencillas, versátiles y en los que podemos controlar con la temperatura y los tiempos de tratamiento, los perfiles de dureza deseados.

\section{Nitruración}

Las observaciones realizadas con el microscopio óptico muestran claramente la existencia de una capa superficial de unos $100 \mu \mathrm{m}$ en la probeta, en la cual la única fase presente es la fase $\alpha$ (Figura 10).

El espesor de la capa observada depende de la temperatura y del tiempo de tratamiento. A mayores temperaturas y tiempos, mayor es el espesor de la capa de fase $\alpha$ observada. La existencia de esta capa demuestra que se ha producido la difusión del nitrógeno gaseoso hacia el interior de la probeta, ya que es el único mecanismo que puede explicar la existencia de la capa superficial de fase $\alpha$ observada (34-37).

Tras examinar las microestructuras, se procedió a determinar los valores de dureza de las probetas tanto en la superficie como en su sección transversal.

Los valores de dureza obtenidos muestran un claro incremento de la dureza superficial con respecto a la dureza de la probeta sin tratar. En algunas probetas se han superado los $1000 \mathrm{HV}_{500}$ de dureza superficial, tal y como puede observarse en la Tabla VIII. En la Figura 11 se muestran los valores de dureza en una sección transversal a diferentes distancias de la superficie de la probeta, para una muestra tratada a $950^{\circ} \mathrm{C}$ durante 8 horas. Se puede observar como la dureza es máxima en la superficie y disminuye hasta los 400 micrómetros de profundidad.

En todas las gráficas se puede comprobar una buena correspondencia entre el valor obtenido de dureza superficial y los valores de dureza a diferentes distancias de la superficie obtenidos en la sección transversal, excepto en los datos de las probetas tratadas a $700^{\circ} \mathrm{C}$. Esta discrepancia puede deberse a que la difusión a la temperatura de 700 ${ }^{\circ} \mathrm{C}$ es muy pequeña, por lo que el nitrógeno no ha penetrado más que unos pocos micrómetros. En

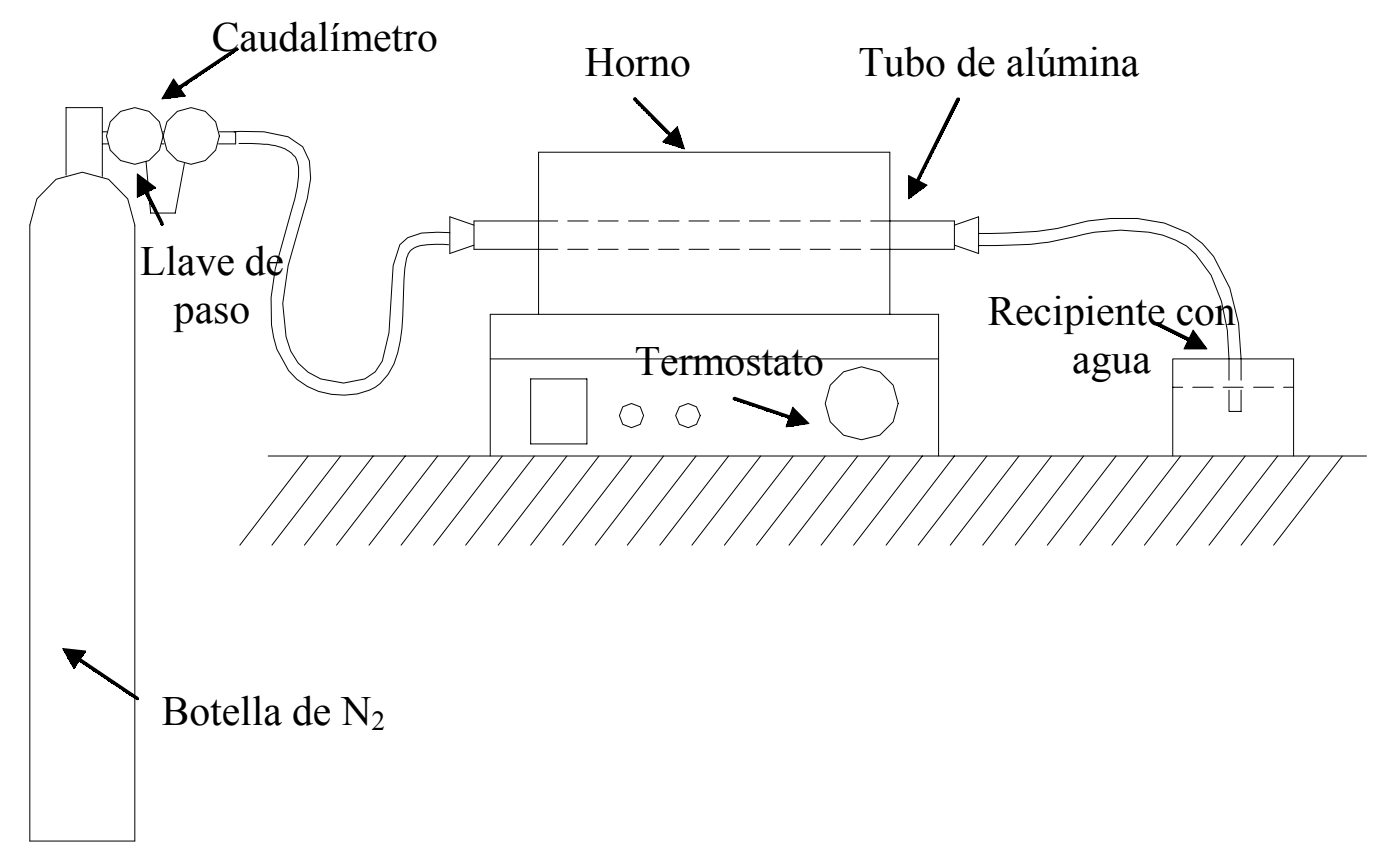

Figura 5. Esquema del horno utilizado. 


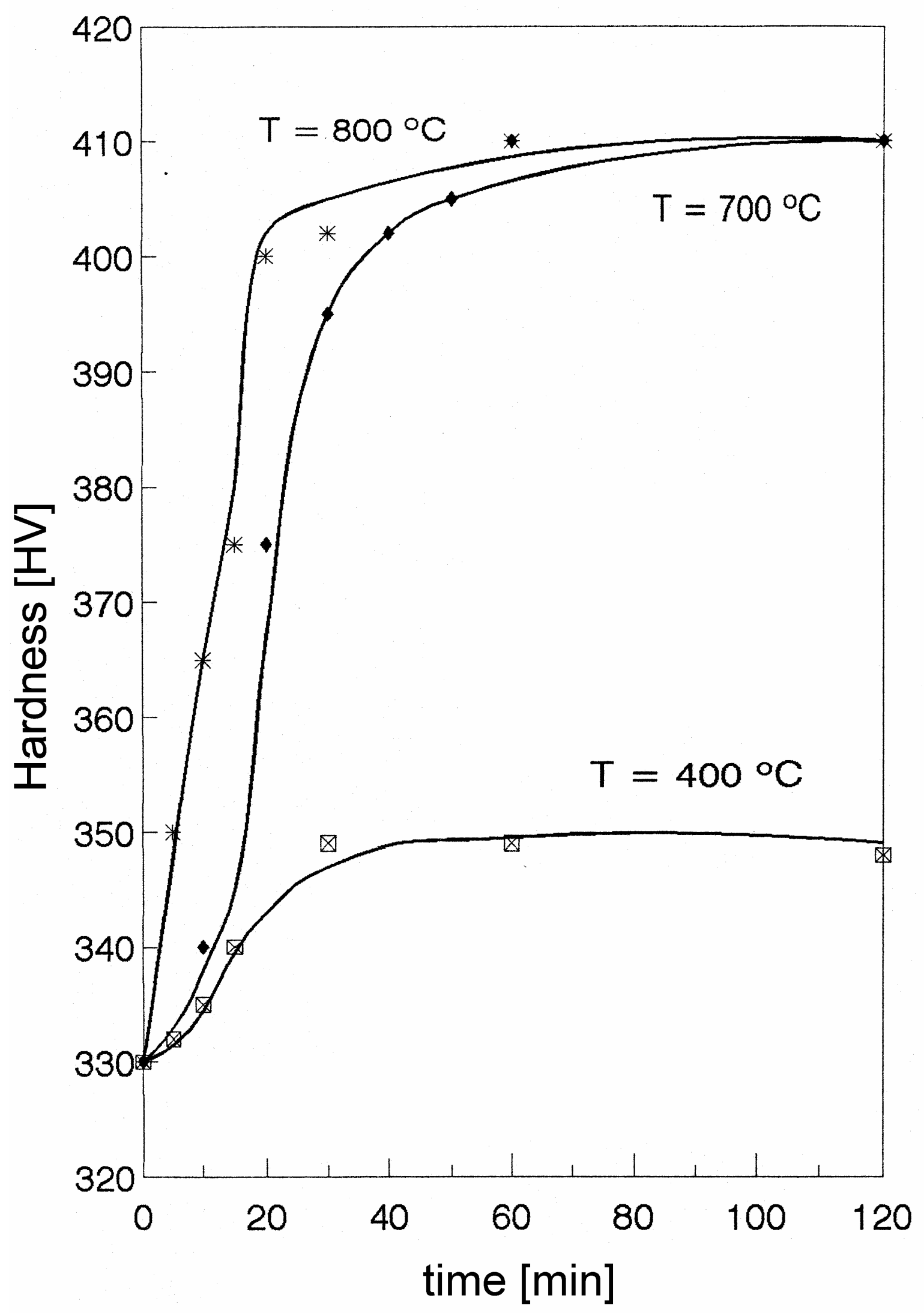

Figura 6. Dureza respecto a las temperaturas de revenido. 


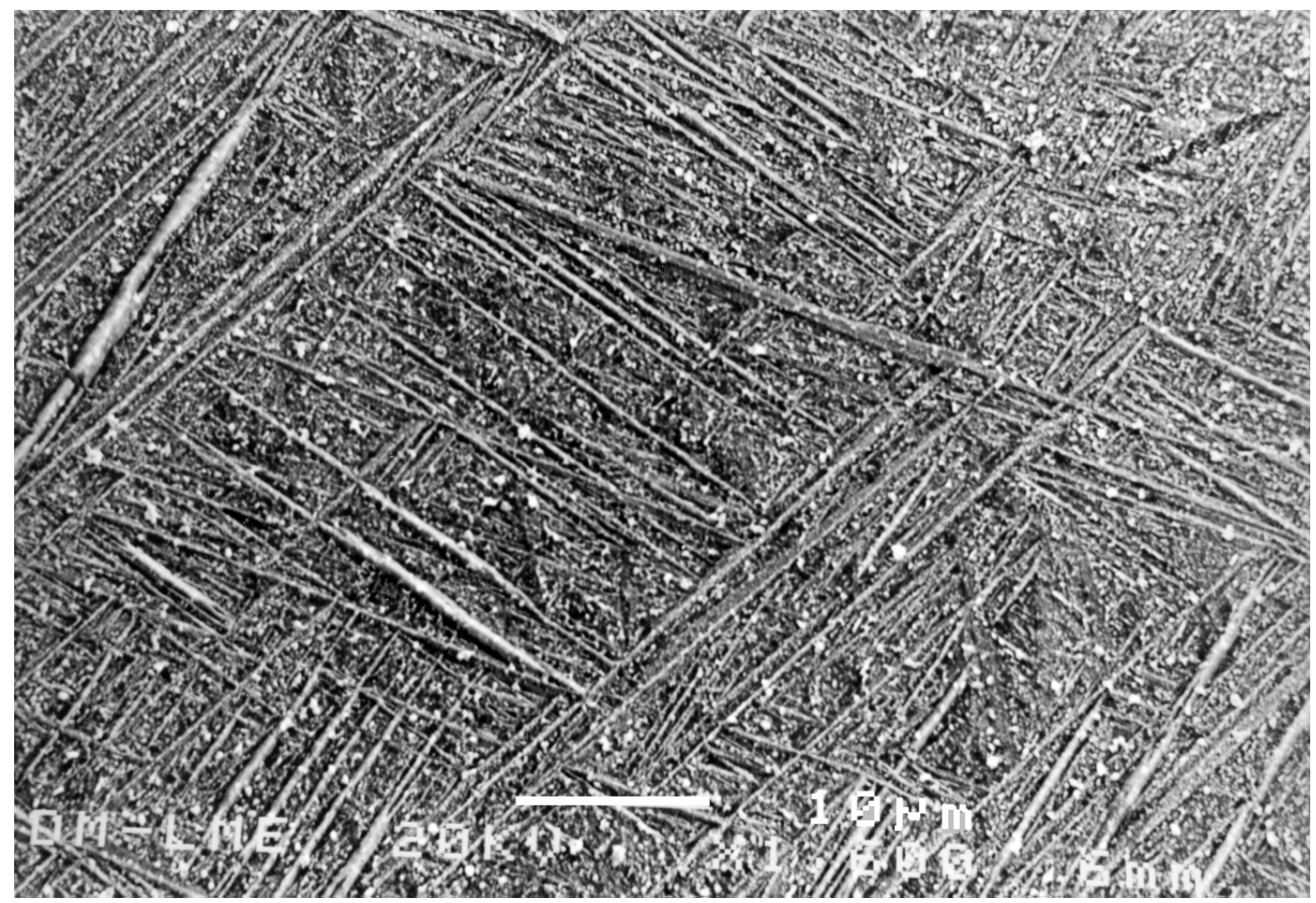

Figura 7. Descomposición de la fase martensítica.

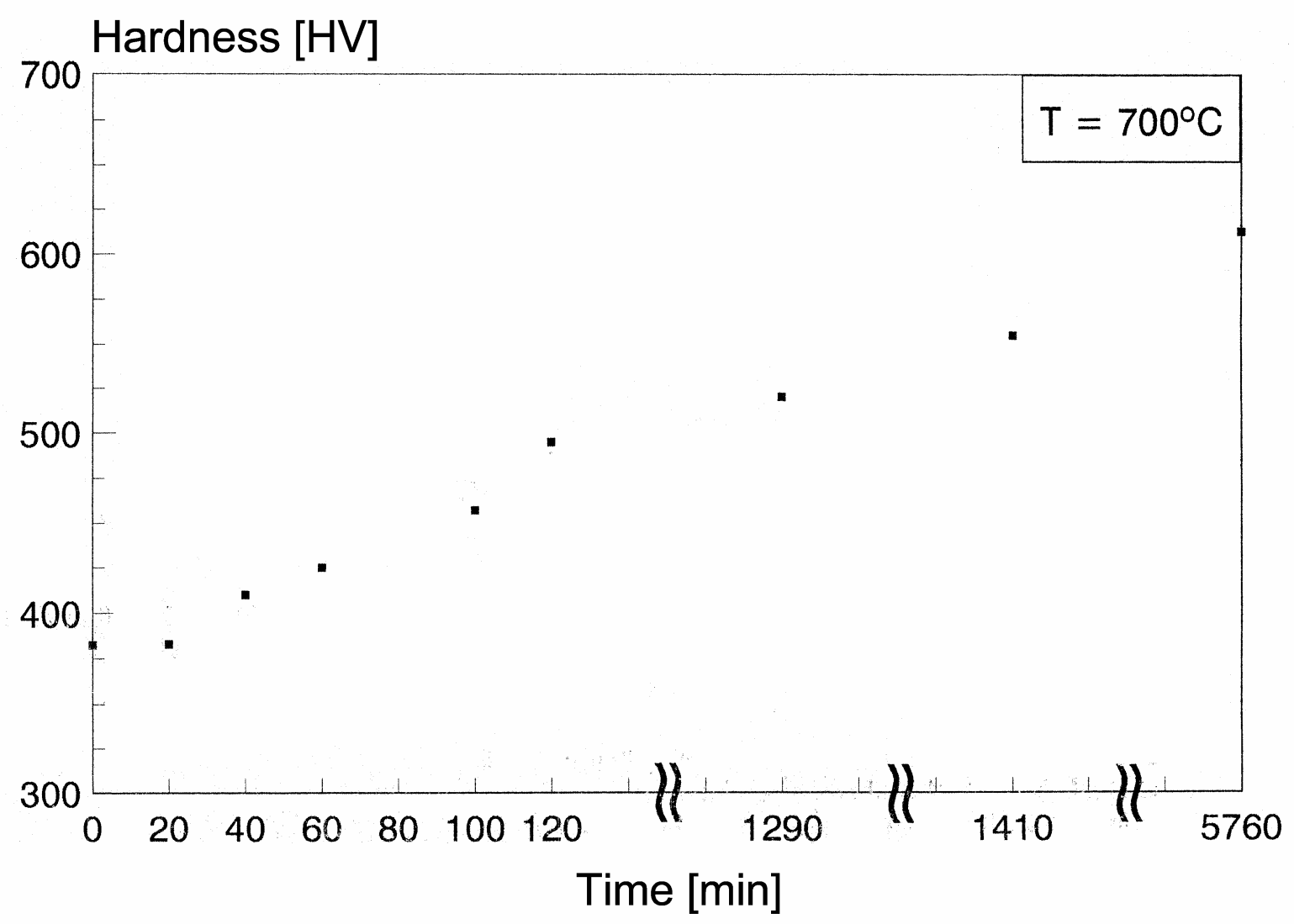

Figura 8. Dureza respecto al revendio y la maduración. 


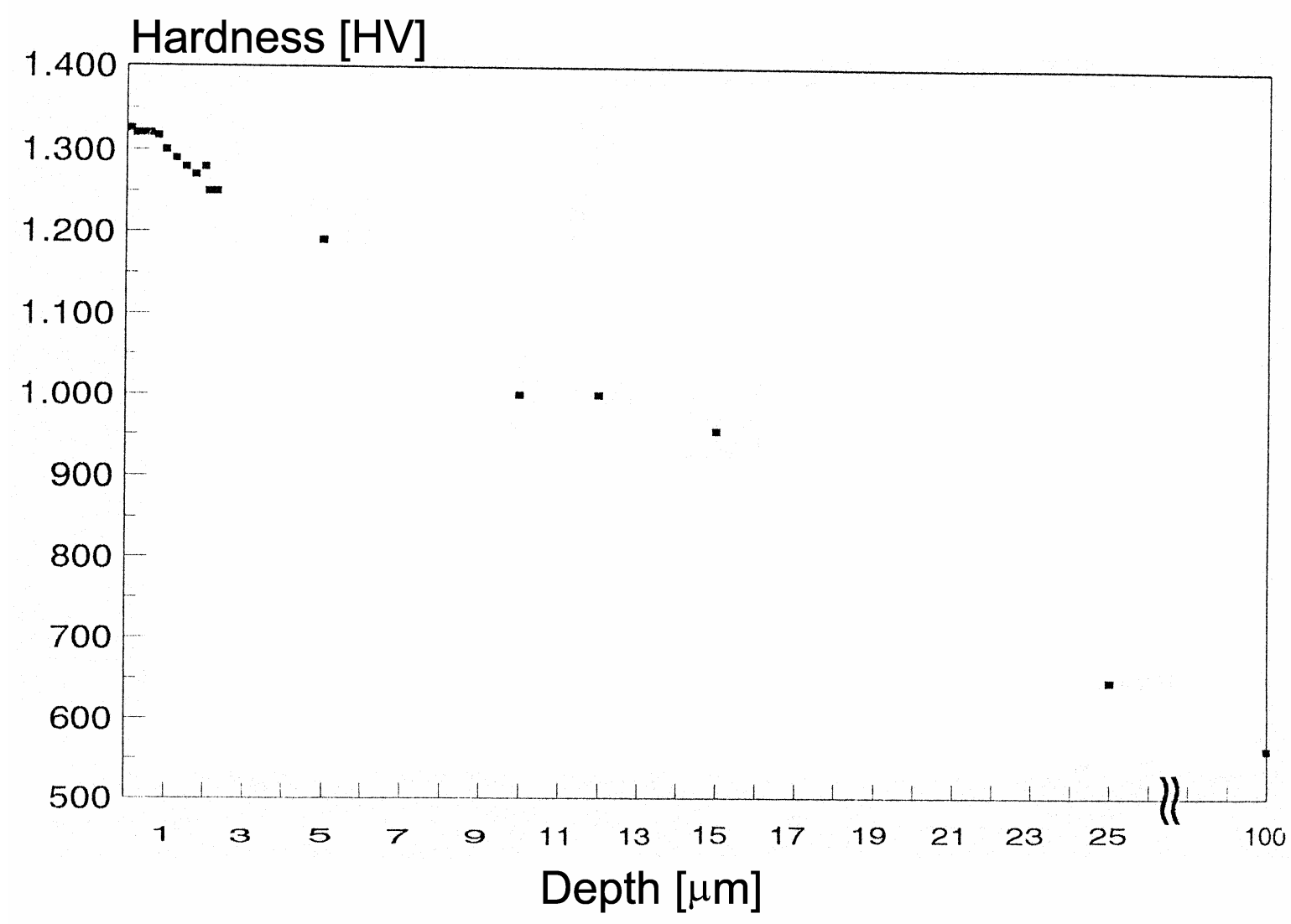

Figura 9. Dureza para los procesos combinados de tratamiento ténmico y anodización para la estructura con fases a, a' y b retenida.
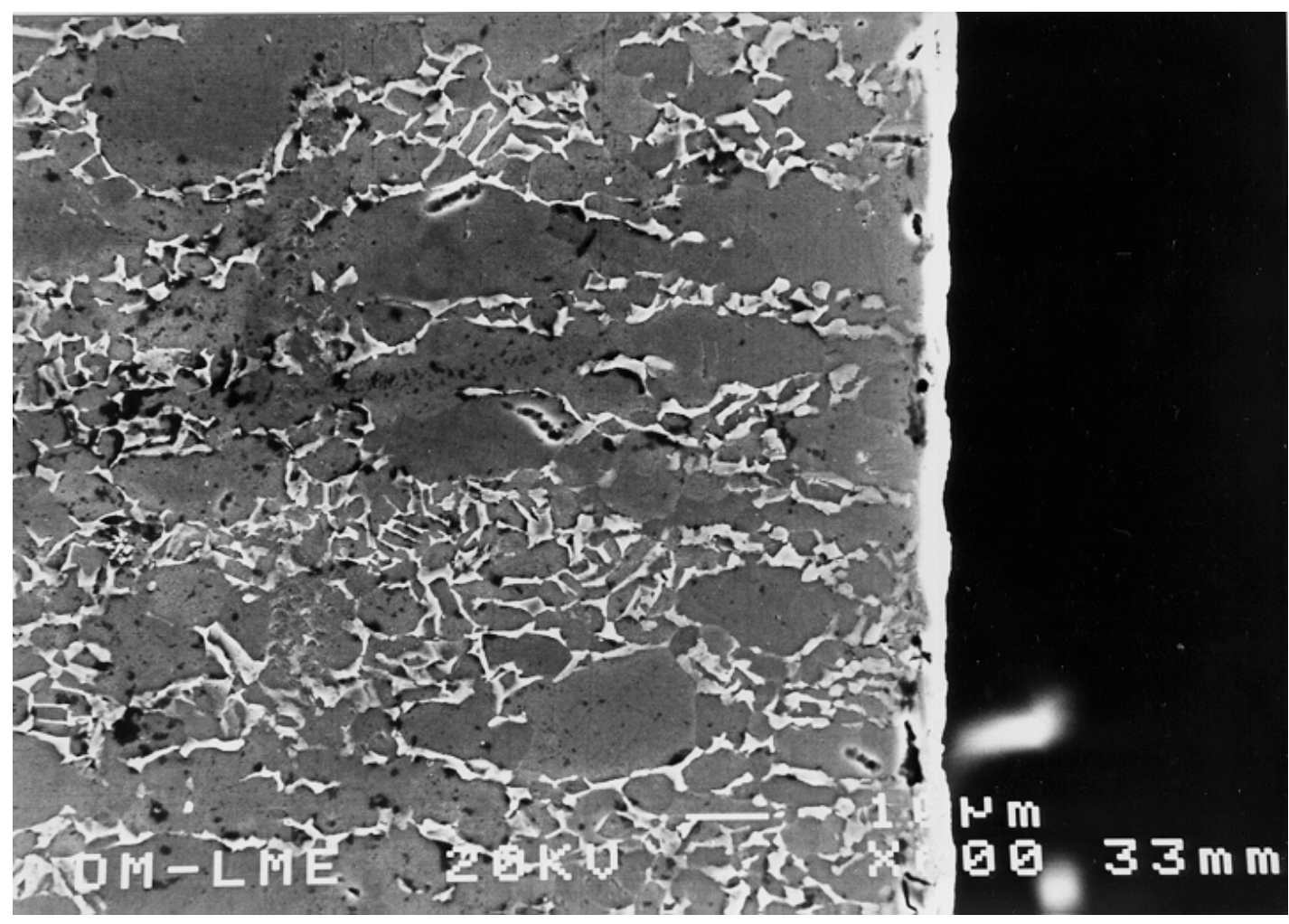

Figura 10. Capa de nitruros y zona de fase a. 


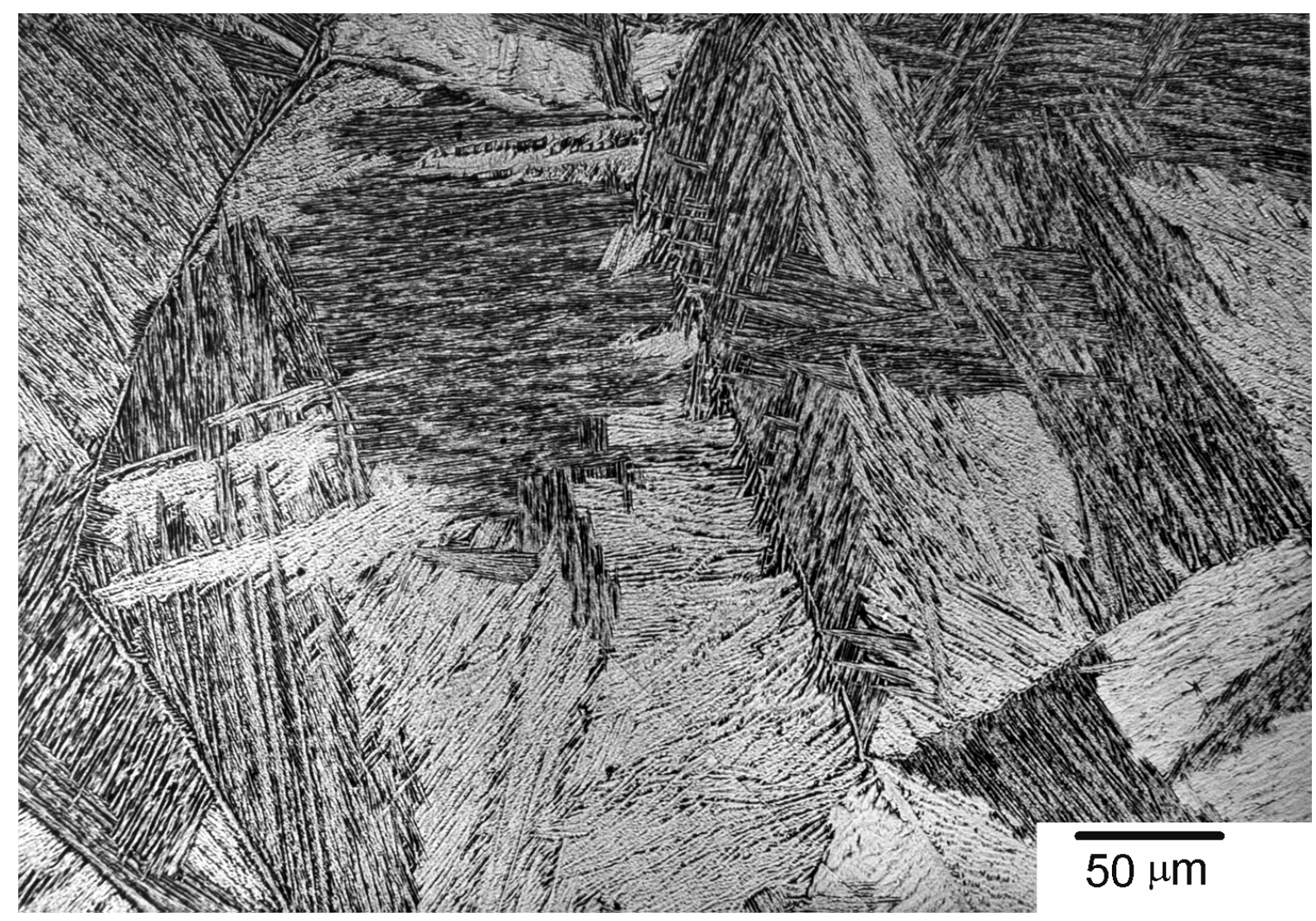

Figura 11. Dureza de la muestra nitrurada respecto a la sección de la muestra.

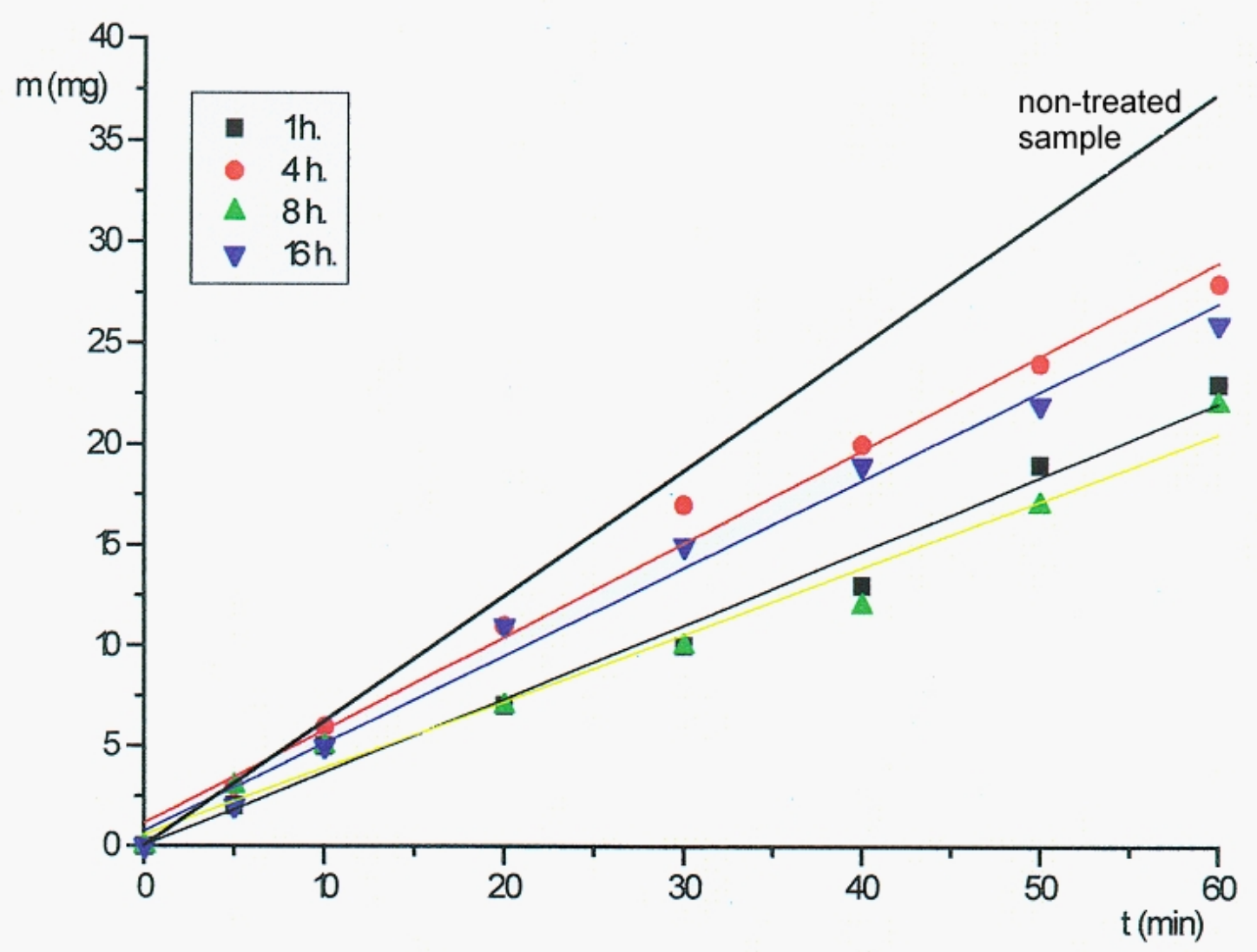

Figura 12. Desgaste de las muestras tratadas a $700^{\circ} \mathrm{C}$ mediante difusión. 


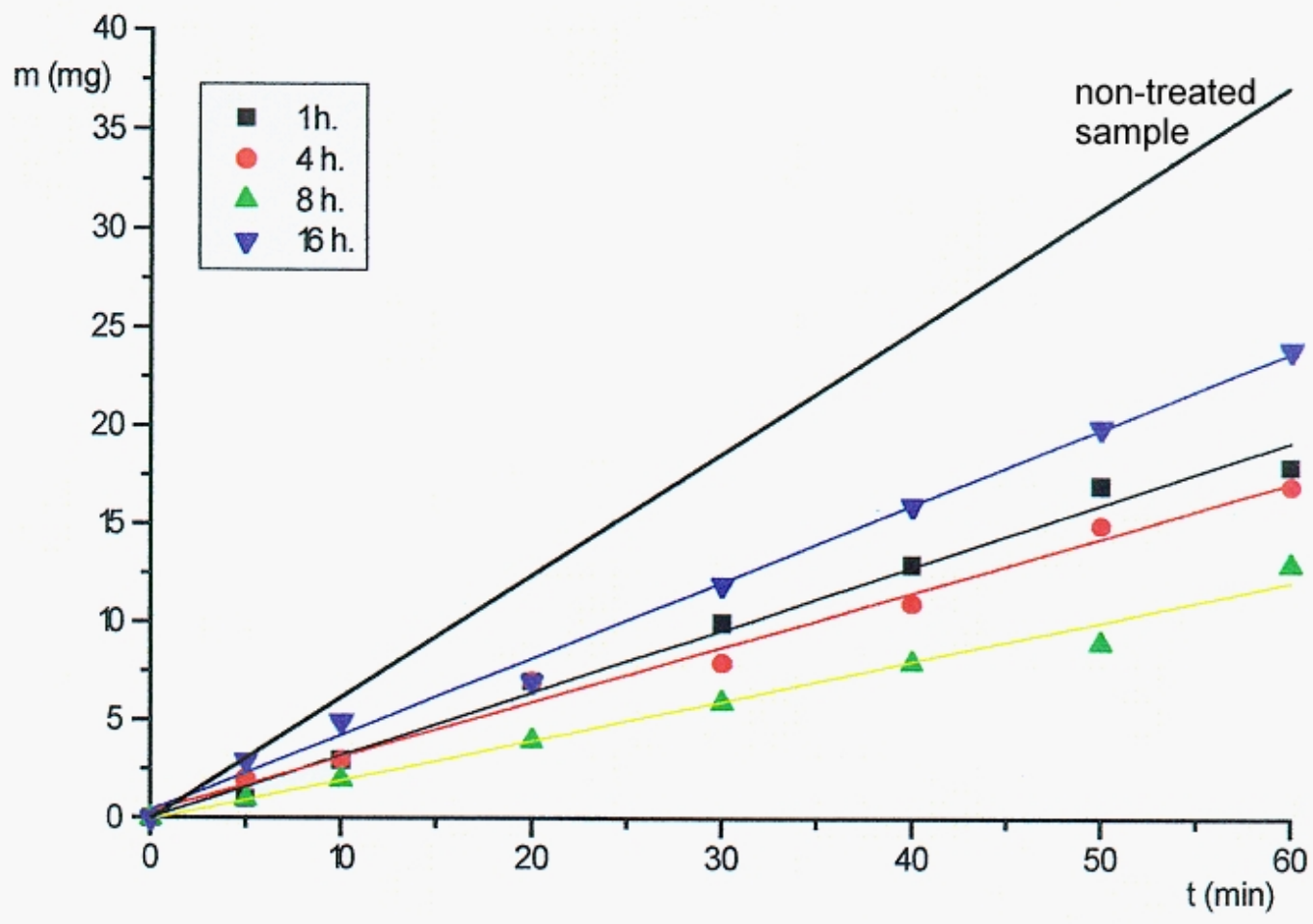

Figura 13. Desgaste de las muestras tratadas a $800^{\circ} \mathrm{C}$ mediante difusión.

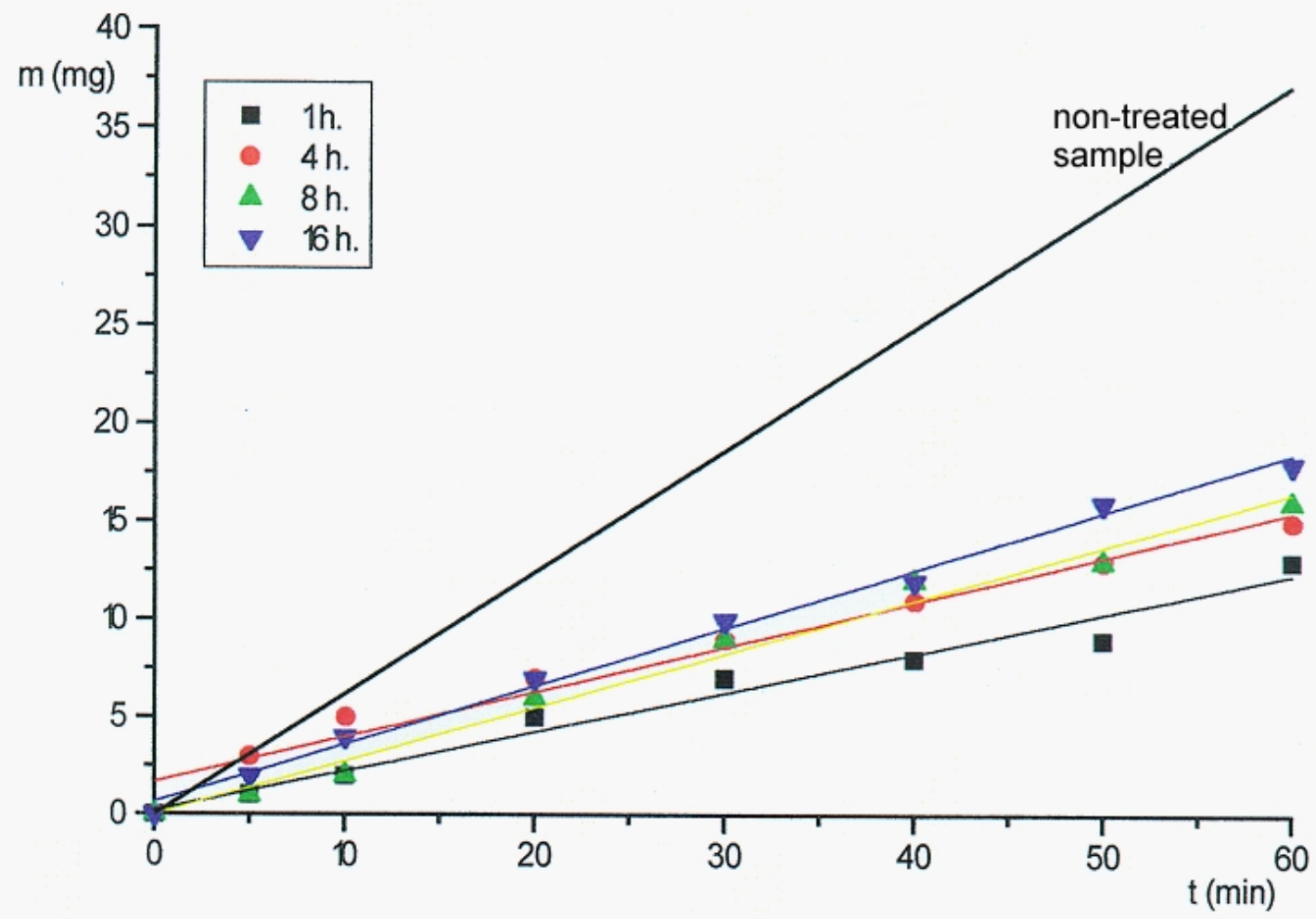

Figura 14. Desgaste de las muestras tratadas a $900^{\circ} \mathrm{C}$ mediante difusión. 
general, la buena correspondencia de valores confirma que no se han producido errores en la medición de la dureza superficial de las probetas (38-39).

Como se ha indicado, se puede observar que la difusión en las probetas tratadas a $700{ }^{\circ} \mathrm{C}$ ha sido muy pequeña, ya que no se aprecia un gran incremento de la dureza superficial. A las temperaturas de 900 y $950{ }^{\circ} \mathrm{C}$, en cambio, el incremento de la dureza superficial es muy importante.
La observación de las gráficas que muestran los valores de dureza para diferentes distancias de la superficie permite realizar una estimación de la profundidad de penetración del nitrógeno en el interior de la probeta, ya que, según la teoría, el incremento de dureza está relacionado con la presencia de nitrógeno en la probeta.

A partir de las gráficas, se ha tomado como límite de la capa nitrurada eficaz aquella distancia de la superficie en la que el valor de dureza cae por debajo de los $500 \mathrm{HV}$, ya que es un valor de

Tabla VII. Dureza $(\mathrm{H})$, energía elástica $\left(\mathrm{W}_{\mathrm{e}}\right)$, energía plástica $\left(\mathrm{W}_{\mathrm{p}}\right)$, energía total $\left(\mathrm{W}_{\mathrm{T}}\right)$ y la relación E/1- $\mathrm{n}^{2}$ ) donde E es el módulo de Young y $\mathrm{n}$ es el coeficiente de Poisson correspondientes a las muestras tratadas a $700^{\circ} \mathrm{C}$ durante diferentes tiempos.

\begin{tabular}{||c|c|c|c|c|c||}
\hline $\begin{array}{c}\text { Tiempo } \\
(\mathrm{min})\end{array}$ & $\mathrm{H}(\mathrm{HV})$ & $\begin{array}{c}\mathrm{W}_{\mathrm{e}} \\
(\mathrm{nJ})\end{array}$ & $\begin{array}{c}\mathrm{W}_{\mathrm{p}} \\
(\mathrm{nJ})\end{array}$ & $\begin{array}{c}\mathrm{W}_{\mathrm{T}} \\
(\mathrm{nJ})\end{array}$ & $\begin{array}{c}\mathrm{E} / 1-v^{2} \\
(\mathrm{GPa})\end{array}$ \\
\hline 60 & 1005 & 0.96 & 1.04 & 2.00 & 232 \\
\hline 120 & 1322 & 1.09 & 1.06 & 2.16 & 229 \\
\hline 600 & 1250 & 0.92 & 0.90 & 1.82 & 258 \\
\hline 1200 & 908 & 0.89 & 1.07 & 1.96 & 250 \\
\hline
\end{tabular}

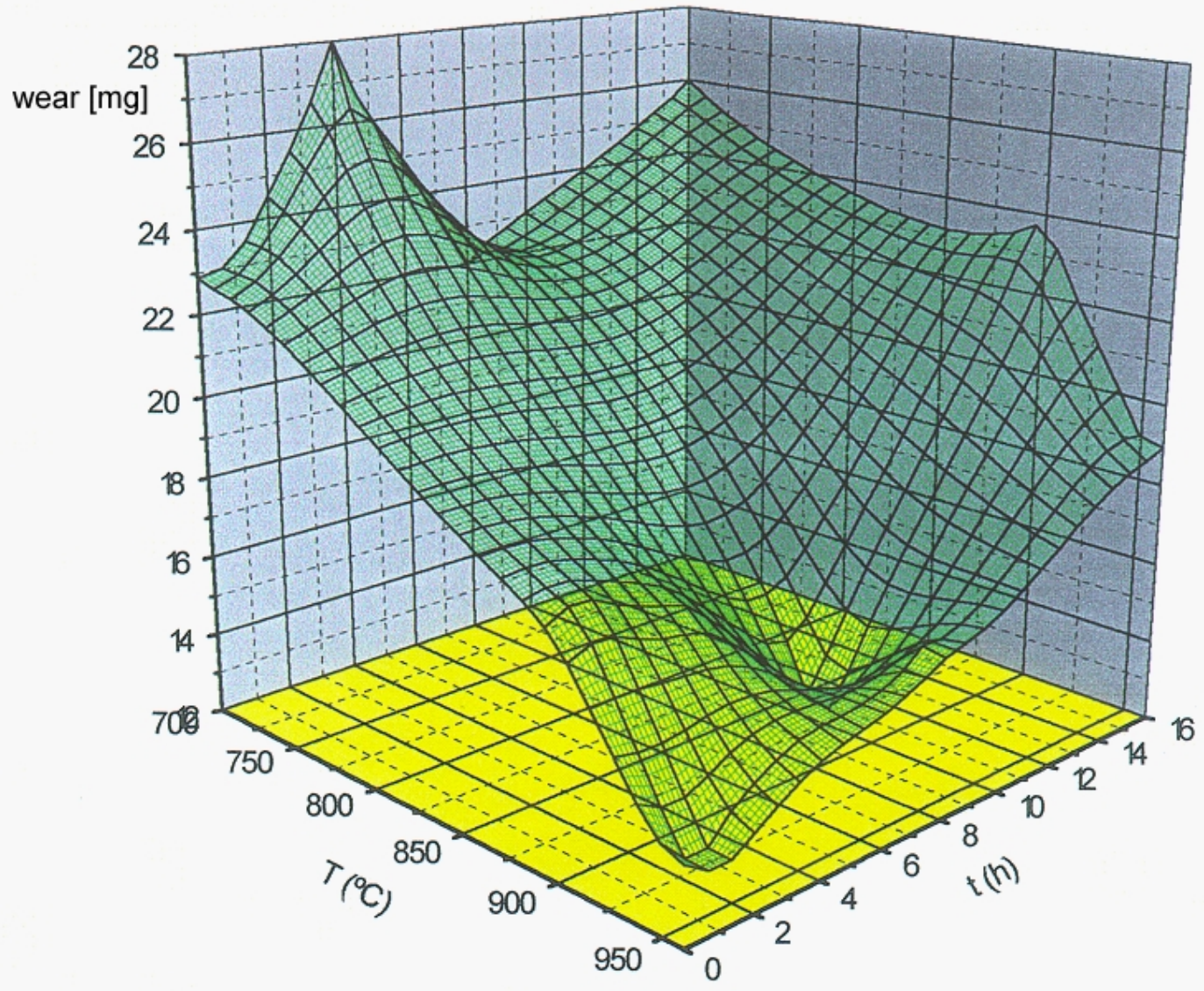

Figura 15. Desgaste de las muestras nitruradas respecto a la temperatura y tiempo de permanencia en el horno. 
dureza no alcanzable mediante el tratamiento térmico del Titanio. Esto se ha tomado como una convención para realizar comparaciones entre probetas, ya que la distancia de penetración del nitrógeno es bastante mayor de lo que indica esta convención.

A partir de esta convención, se observa que, en término medio, el grosor de la capa nitrurada eficaz se sitúa en torno a los $60100 \mu \mathrm{m}$. Si se realiza un gráfico con los espesores de capa nitrurada versus la temperatura y el tiempo de tratamiento, se comprueba la fuerte relación que existe, ya que a mayores temperaturas y/o tiempos, el grosor de la capa nitrurada aumenta, tal y como cabe esperar por las características del proceso de difusión del nitrógeno.

Tras observar la microestructura y realizar las mediciones de dureza de las probetas se procedió a realizar las pruebas de desgaste abrasivo, a fin de comprobar la efectividad del tratamiento de nitruración gaseosa en la reducción del desgaste.

Para cada ensayo de una probeta, se obtuvieron 7 valores de desgaste en un intervalo de 60 minutos. Esto permite comprobar que no existe ningún problema que afecte al ensayo, así como realizar un seguimiento del desgaste durante todo el ensayo. En las Figuras 12, 13 y 14 se representan las curvas de desgaste de las 12 probetas ensayadas, y se comparan con los resultados obtenidos de los ensayos de desgaste de una probeta sin tratar.

Los datos de desgaste obtenidos de los experimentos muestran reducciones de más de $2 / 3$ en el desgaste sufrido por las probetas tratadas mediante nitruración gaseosa, en comparación con la probeta sin tratar. Esto permite decir que el tratamiento es válido para obtener una disminución del desgaste en las piezas de Ti.

Si se representan a la vez los valores de temperatura y tiempo de tratamiento y el desgaste total sufrido durante el ensayo de desgaste por la probeta, se puede comprobar que, si bien en líneas generales a una mayor dureza le corresponde un menor desgaste, el valor mínimo de desgaste no se encuentra en el punto de mayor temperatura y tiempo de tratamiento, sino en un punto intermedio.

Este hecho puede deberse al efecto de aumento de fragilidad que produce el nitrógeno cuando el porcentaje de composición del nitrógeno en la aleación Titanio aumenta excesivamente. Con esta hipótesis se explica el hecho de que, si bien la dureza es mayor para los tratamientos realizados a las máximas condiciones de temperatura y tiempo, la disminución del desgaste no siga la teoría de desgaste, sino que presente un mínimo de silla en la superficie de la Figura 15.

El mínimo valor de desgaste se halla entre un tratamiento a $900^{\circ} \mathrm{C}$ durante 8 horas, y un tratamiento a $950^{\circ} \mathrm{C}$ durante 1 hora, tal y como se observa en la Figura 15

Esta disminución del desgaste permite que este tratamiento de endurecimiento sea útil en aplicaciones que, sin sufrir un desgaste constante, requieran una protección suplementaria contra el desgaste, o bien en aplicaciones donde las cargas sean ligeras o medianas.

\section{Agradecimientos}

Los autores desean agradecer a Klockner la donación del material para este estudio y en especial a la Sra. Mercedes Roldán Chesa su ánimo y ayuda para seguir investigando por la implantología

\section{Bibliografía}

1. Semlitsch,M. Staub. S. and Weber H. Titanium aluminium niobiun alloy, development for biocompatible, high Strength surgical implants. Biomed. Technik 1989, 334339.

2. H.P.Tümmler, R.Thull and M.Schaldach. The mechanism of repassivatión and the concentration of corrosion products shown on Ti Al V. World Congress en Medical Physics and Biomedical Engineering. Hamburg, 1982 paper 3.11 .

3. J.O.Galante, W.Rostoker. Wear in total hip prostheses. Acta Orthop.Scand. Suppl.145 (1973), 146.

4. R.M.Streicher, R.Schoen. Tribology in medicine. Proceedings of the 1st implant Material Congress. Frankfurt (1989).

Tabla VIII. Valores de dureza superficial de las probetas tratadas, en $\mathrm{HV}_{500}$.

\begin{tabular}{|c||c|c|c|c|}
\hline \multicolumn{1}{|c||}{} & \multicolumn{4}{c|}{$\mathrm{t}[\mathrm{h}]$} \\
\hline $\mathrm{T}\left[{ }^{\circ} \mathrm{C}\right]$ & 1 & 4 & 8 & 16 \\
\hline \hline 700 & 413 & 544 & 717 & 547 \\
\hline 900 & 841 & 895 & 928 & 1074 \\
\hline 950 & 1018 & 1028 & 967 & 1097 \\
\hline
\end{tabular}


5. A.Yamagami, Y.Hishio and Y.Yoshiara. Influence of surface topology of titanium implant on fixation in the bone. Fourth World Biomaterials Congress. Berlin. (1992) paper 386.

6. A.Cigada, M.Cabrini and P.Pedeferri. Increasing of the corrosion resistance of the Ti-6Al-4V alloy by high thickness anodic oxidation. J. of Mater. Sci: Materials in Medicine 3 (1992) 408-412.

7. T.Bell European J. Engineeering Education 12 (1987) 27-32.

8. B.Finern. Surfaces résistant à l'usure sur le titane et les alliages de titane et obtenues par un traitement en bains de sels. Harterei Technische Mitteilungen 26(1971) 47.

9. J.L.Wyatt y N.J.Grant. Nitruration du titane par l'ammoniac. Trans ASM 46 (1978) 540-567.

10. B.Finern. Surfaces résistant à l'usure sur le titane et les alliages de titane et obtenues par un traitement en bains de sels. Harterei Technische Mitteilungen 26(1971) 52 .

11. J.Lanagan, P.M.Morton y T.Bell. Traitment de surface du titane avec un plasma à décharge luminescente. Designing with titanium. (1986) Institute of Metals. 136-150.

12. J.Lanagan. VIth Titanium World Congress. Ed. Physique (1989) T4. 1957.

13. I.S.Polkin y B.A.Kolachev. Transformation de phases, traitements thermiques et thermomécaniques pour les alliages de titane. Traitement Thermique 241 (1990) 35-59.

14. I.J.Polmear. Light alloys. Edward Arnold. Londres (1981).

15. E.W.Collings. The physical metallurgy of Titanium alloys. American Society for Metals. Ohio (1984).

16. P.Tarín. Titanio y sus aleaciones. CEAM. Barcelona(1991).

17. G.Willems, J.P.Celis, P.Lambrechts, M.Braem y G.Vanherle. Hardness and Young's modulus determined by nanoindentation technique of filler particles of dental restorative materials compared with human enamel. J. of Biomed. Mater. Research. 27 (1993) 747-755.

18. H.Zitter y H.PLenk. J.Biomed. Mater. Res. 21 (1987) 881.

19. S.Nasser, P.Campbell y H.C.Amstutz. Metals and their alloys in orthopaedic surgery. Ohio. American Society for Metals. (1989).

20. B.A.Kolachev. Metallographie des alliages de titane. Ed. Glazunov Metallurgiya. (1980) 464.

21. Y.Murakami, K.Nakao y V.Yasuda. Titane, Science et Technologie. Oberursel. (1985) 1543.

22. J.A. Garcia Poggio, P.Tarín y E.Suárez. Estudio de las transformaciones estructurales de diversas aleaciones de Titanio. VI Asamblea General del CENIM(1985). 9.
23. P.Tarín y I.Goñi. Estudio metalográfico y de las transformaciones en titanio y aleaciones mediante técnicas de análisis térmico. V Asamblea General del CENIM. (1981) 124.

24. F.J.Gil, J.M.Manero, M.Marsal, N.Salán y J.A.Planell. Estudio de la microestructura de martensita revenida a $500^{\circ} \mathrm{C}$ en la aleación Ti-6Al4V. Coloquio Franco-Ibérico de Microscopía Electrónica. ED. UB. (1991) 276-277.

25. F.J.Gil, E.Fernández, R.Arcas y J.A.Planell. Endurecimiento superficial mediante tratamientos térmicos y anodizado de la aleación Ti-6Al-4V para implantes quirúrgicos. Biomecánica 2. en prensa.

26. J.M.Donachie. Titanium: a Technical Guide. American Society for Metals. Ohio. 1988.

27. JC.Williams, A.F.Belov. Titanium and Titanium alloys. Proceedings of Third International Conference on Titanium. Plenum Press. Nueva York (1982).

28. H.Kimura, O.Izumi. Titanium' 80 Science and Technology. Proceedings of Fourth International Conference in Titanium. Kyoto (1980) Met.Soc.AIME. Warrendale PA (1980).

29. H.Conrad y R.Jones. Effects of interstitial content and grain size on the mechanical behaviour of alpha titanium below 0.4Tm. Proc. First Int.Conf. on Titanium. Londres. Pergamon Press (1970).

30. H.Conrad, B.de Meester, M.Doner y K.Okasaki. Strengthening of alpha titanium by the interstitial solutes. J. Less Common Metals 39 (1975) 1-45.

31. H.Conrad. Effect of interstitial solutes on the strngth and ductility of Titanium. Pogr. Mater. Sci. 26 (1981) 123-203.

32. P.H.Morton y T.Bell. Surface engineering with titanium. Mémoires et études scientifiques. Revue de Métallurgie. (1989) 639-646.

33. A.K.Suvi, R.N.nimmagadda y R.F.Bunshah. Influence of ion implantation and overlay coatings on various physico-mechanical and wear properties of stainless steel, titanium and aluminium. Thin Solid. Films. 64 (1979) 191-203.

34. Annual Book of ASM Standards, vol. 13. American Society for Metals. Easton, EEUU,1994.

35. Donachie, M. Titanium, A Technical Guide. American Society for Metals Ohio.1988.

36. Gil F.J., Fernández E. Endurecimiento superficial mediante tratamientos térmicos de la aleación Ti 6Al 4V para implantes quirúrgicos. Biomecánica. 2,(1993) 51.

37. Gilbert S. Heat treating of Titanium and Titanium Alloys. Mat Sci Rev, (1992).35-37

38. Kossowsky, R. Surface modification engineering (vol. 1). Boca Raton (EEUU), (1984).

39. Polmear, I.J. Light Alloys. Metallurgy of the Light Metals. De. Arnold. London. (1999). 